\title{
Comment modifier la forme des cellules en exprimant un gène codant pour une protéine du cytosquelette?
}

Bien que l'on suppose depuis longtemps que les protéines du cytosquelette aient un rôle dans la morphogenèse cellulaire, il n'y a, à l'heure actuelle, que peu d'études démontrant cette hypothèse.

Lors de la différenciation des cellules intestinales du type absorptif, de nombreuses digitations qui constituent la bordure en brosse se forment au pôle apical de ces cellules. Dans cet organite cellulaire, on distingue deux régions : les microvillosités, qui contiennent un faisceau axial d'actine connecté latéralement à la membrane plasmique par des ponts protéiques, et une ceinture apicale de microfilaments (terminal web) orientée perpendiculairement à l'axe des microvillosités dans lequel plongent les faisceaux d'actine qui supportent la membrane des microvillosités [9]. Le haut degré d'organisation du cytosquelette de la bordure en brosse et le fait que l'on puisse l'isoler aisément en font un modèle particulièrement intéressant pour étudier les interactions moléculaires entre la membrane et les éléments du cytosquelette $[5,7]$.

Les études biochimiques et morphologiques ont suggéré que la villine, une des protéines majoritaires associées aux microfilaments d'actine, joue un rôle capital lors de l'assemblage de cette structure spécialisée de la surface cellulaire.

In vitro, la villine modifie, en fonction de la concentration en calcium, la longueur des filaments d'actine $F$ ou provoque leur association en faisceaux. Contrairement aux autres pro- une spécificité tissulaire : on ne la trouve que dans les cellules ayant une bordure en brosse fonctionnelle ou dans des cellules de même origine embryonnaire $[6,8]$. Les études sur des entérocytes humains en culture, celles faites chez l'homme adulte et celles faites sur des organismes en développement, ont montré qu'il y avait une forte augmentation de la synthèse de villine juste avant l'assemblage de la bordure en brosse. Parallèlement à l'augmentation de la quantité de villine synthétisée, on observe une localisation préférentielle sous la membrane plasmique apicale $[3,8]$.

Le clonage de l'ADNc codant pour la villine humaine [1] a ouvert la possibilité de développer un modèle utilisable pour analyser le rôle joué par cette protéine dans l'organisation du cytosquelette et de la surface cellulaire. Des fibroblastes de rein de singe (lignée CV1) qui normalement n'expriment pas la villine ont été transfectés avec l'ADNc codant pour la villine humaine inséré dans un vecteur d'expression capable d'en assurer la production dans ces cellules. On a pu montrer que l'expression de la villine seule est suffisante pour provoquer un profond changement de l'organisation cellulaire [4]. L'analyse par microscopie à balayage de la surface cellulaire des cellules transfectées a révélé une augmentation du nombre et de la longueur des microvillosités (figure 1a et 1b).

Par double marquage en immunofluorescence de la villine et de l'actine filamenteuse, on a démontré une réorganisation des microfilaments d'actine. Les microvillosités qui apparaissent à la surface cellulaire sont soutenues par un faisceau de filaments d'actine auquel est associée la villine. Dans les mêmes cellules, on observe fréquemment une dislocation des câbles d'actine, normalement présents dans les fibroblastes en culture. Ces observations démontrent que, in vivo, la villine induit une redistribution de l'actine filamenteuse entraînant des changements de la surface cellulaire. Ces modifications n'ont lieu que dans les cellules ayant un niveau élevé de villine et indiquent qu'il existe un seuil de concentration en-dessous duquel la villine n'induit pas ce changement morphologique.

Figure 1. La transfection des cellu- $\triangleright$ les CV1 avec le vecteur PSV51, dans lequel on a inséré I'ADNc complet codant pour la villine, induit la formation de nombreuses et longues microvillosités sur la surface dorsale des fibroblastes. 1a. Sur la surface des cellules contrôle, il y a des microvillosités courtes et très peu nombreuses. 1b. La surface des cellules transfectées est couverte de nombreuses microvillosités très longues et rigides Imicroscopie électronique à balayage; barre : 1 $\mu \mathrm{m})$. 1c. En immunofluoresence, avec des anticorps antivilline, on met en évidence la présence de cette protéine dans les microvillosités induites par l'expression du gène de la villine. 

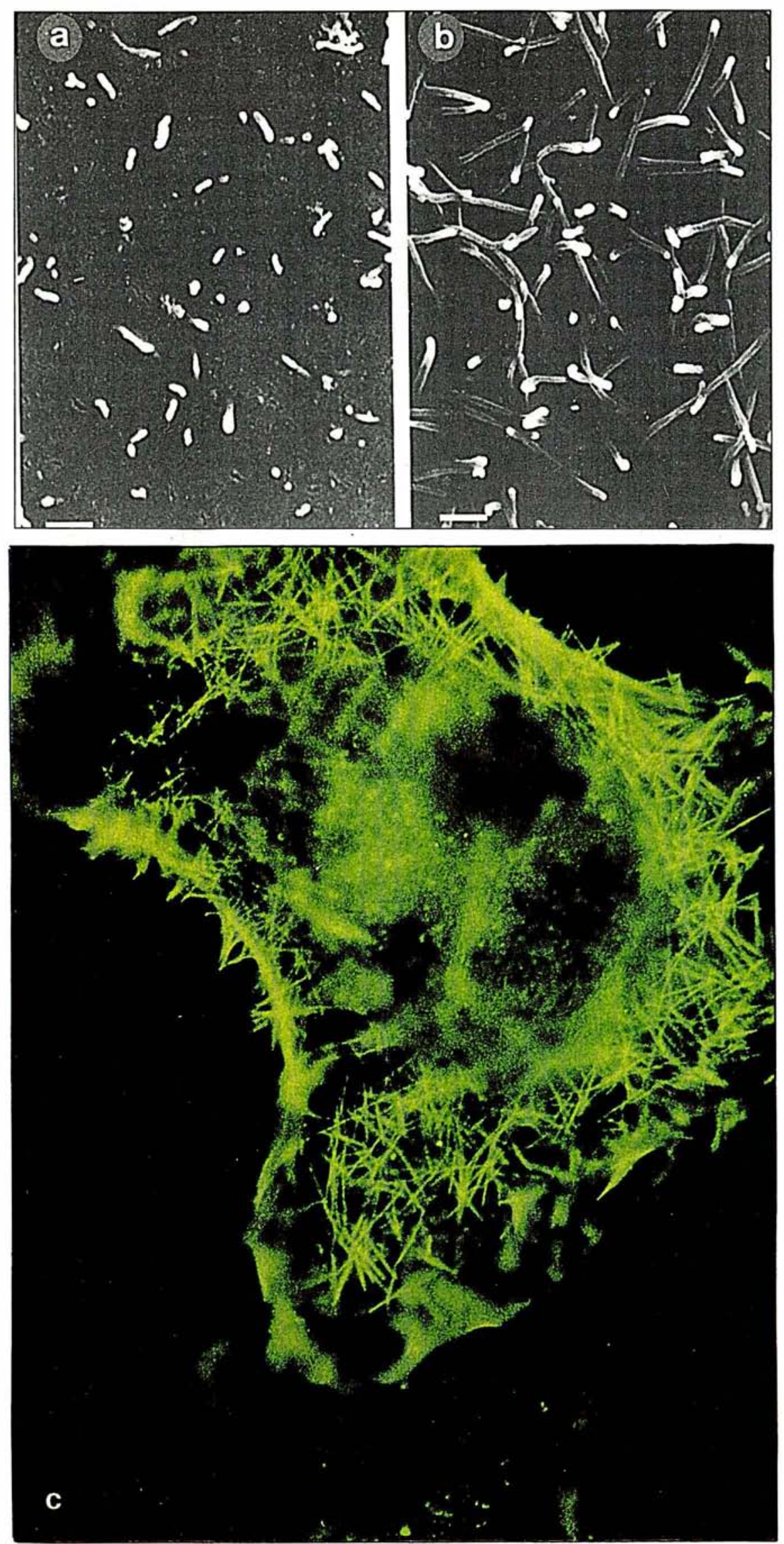

$\mathrm{m} / \mathrm{s} n^{\circ} 5 \mathrm{vol}$. 6, mai 90
L'analyse de la séquence de l'ADNc des villines humaine et de poulet a montré que leur structure primaire est hautement conservée. L'étude de l'organisation moléculaire de la villine a révélé deux régions principales: un grand domaine de $90 \mathrm{kDa}$ appelé "noyau" (core) et un petit domaine de $8,5 \mathrm{kDa}$, situé dans la région carboxyterminale, baptisé la "tête" (headpiece). Le " noyau " de la villine est analogue à d'autres protéines liant l'actine, mais la "tête " est en revanche spécifique. Des expériences in vitro avec des fragments protéolytiques de la villine ont montré que la présence de la "tête " est nécessaire pour provoquer l'association des filaments d'active en faisceaux. L'expression d'un ADNc "villine mutant" tronqué de la séquence codant pour la "tête" a permis de montrer que ce domaine est essentiel pour provoquer les modifications de la surface cellulaire et la réorganisation du cytosquelette dans les fibroblastes transfectés.

La conclusion de ces expériences est que l'expression du gène codant pour la villine est suffisante pour induire la morphogenèse de microvillosités dont l'assemblage implique des interactions spécifiques entre le cytosquelette et la membrane plasmique. De plus, les résultats confirment directement le rôle essentiel joué par la villine dans l'assemblage de la bordure en brosse des cellules épithéliales et suggèrent que les activités de la villine sur la polymérisation de l'actine observées in vitro ont également lieu in vivo.

Nos résultats montrent aussi que la " tête " de la villine joue un rôle fondamental dans les interactions actinevilline in vivo. Le fait qu'il y ait dans la molécule un domaine spécifique indispensable, mais pas nécessairement suffisant, pour induire la croissance des microvillosités, est un argument supplémentaire pour conclure que la villine, dont l'expression est spécifique du tissu, a un rôle distinct de celui des autres protéines liant l'actine qui sont largement exprimées dans de nombreux tissus.

Les microvillosités qui ont été induites par la villine dans les fibroblastes non polarisés sont moins rigides et moins régulières que celles de la 
bordure en brosse de l'entérocyte. Cela est probablement dû à l'absence de facteurs habituellement présents dans les cellules épithéliales polarisées qui forment une bordure en brosse. Récemment, on a pu reconstituer in vitro, en ajoutant à des microfilaments d'actine $\mathrm{F}$ les trois protéines majoritaires de la bordure en brosse (villine, fibrine, myosine I de la bordure en brosse), des faisceaux d'actine structurellement semblables à ceux constituant le cytosquelette des microvillosités in vivo [2]. Lorsqu'ils seront disponibles, il sera envisageable de cotransfecter des cellules en culture avec les $\mathrm{ADNc}$ codant pour ces trois protéines et d'étudier ainsi leur fonction. Pour évaluer l'influence des facteurs cytosoliques spécifiques de certains types cellulaires, il sera également intéressant d'essayer d'exprimer le gène de la villine dans des cellules épithéliales polarisées, mais qui n'expriment pas de villine et qui ne forment pas de bordure en brosse.

On peut aussi imaginer que la synthèse d'une protéine capable d'induire des changements de la surface et de l'organisation intracellulaire puisse modifier globalement la machinerie cellulaire ainsi que le programme génétique. Il serait important, par exemple, d'étudier si la présence de la villine entraîne dans les fibroblastes transfectés l'expression de gènes qui sont impliqués dans le programme de différenciation des cellules épithéliales.

Cette approche expérimentale généralisée à d'autres systèmes devrait permettre d'étudier in vivo la fonction des protéines qui, comme la villine, sont associées au cytosquelette et ont un rôle dans l'organisation cellulaire.

\section{E.F. \\ C.H. \\ D.L.}

\begin{abstract}
1. Arpin M, Pringault E, Finidori J, et al. Sequence of human villin : a large duplicated domain homologous with other actinsevering proteins and a unique small carboxy-terminal domain related to villin specificity. J Cell Biol 1988; 107 : 1759-66. 2. Coluccio LM, Bretscher A. Reassociation of microvillar core proteins : making a microvillar core in vitro. J Cell Biol 1989 . 108 : 495-502.
\end{abstract}

3. Dudouet B, Robine S, Huet C, et al. Changes in villin synthesis and subcellular distribution during intestinal differentiation of HT29-18 clones. J Cell Biol 1987 ; 105 : 359-69.

4. Friederich E, Huet C, Arpin M, Louvard $\mathrm{D}$. Villin, induces microvilli growth and actin redistribution in transfectedfibroblasts. Cell 1989; 59 : 4 61-75.

5. Louvard D. 1989. The function of the major cytoskeletal components of the brush border. Curr Op Cell Biol 1989 ; 1: 51-7. 6. Maunoury R, Robine S, Pringault E, $e$ al. Villin expression in the visceral endoderm and in the gut anlage during early mouse embryogenesis. EMBO J, 1988; 7 : 3321-9.

7. Mooseker MS. Organization, chemistry, and assembly of the cytoskeletal apparatus of the intestinal brush border. Ann Rev Cell Biol 1985 ; 1 : 209-41.

8. Robine S, Huet C, Mol R, et al. Can villin be used to identify malignant and undifferentiated normal digestive epithelial cells? Proc Natl Acad Sci USA 1985; 82 : 8488-92.

9. Robine S, Dudouet B, Louvard D. La villine, un marqueur de différenciation utilisé en cancérologie. médecine/sciences 1987 ; 3 : 479-86.

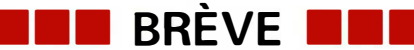

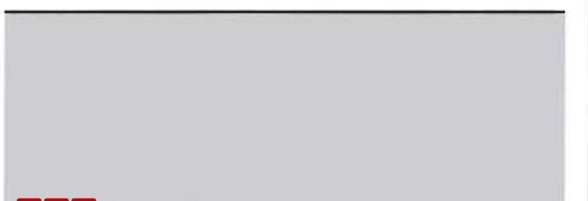

L'adipsine est un marqueur secondaire de l'obésité. Nous avons rapporté dans $\mathrm{m} / \mathrm{s}$ que l'adipsine, une sérine protéase appartenant au système du complément $\left(\mathrm{m} / \mathrm{s} n^{\circ} 8\right.$, vol. 5, p. 601) était abaissée dans les obésités génétiques $\left(\mathrm{m} / \mathrm{s} n^{\circ} 9, \mathrm{vol}\right.$. 3, p. 555 et 557), et pouvait donc peutêtre servir de marqueur prédictif dc ces anomalies. En fait, unc équipe parisienne dirigée par M. Lavau vient de démontrer que, dans un modèlc murin de diabète et d'obésité génétiques (souris $\mathrm{db} / \mathrm{db}$ ), la diminution de l'expression de l'adipsine et de son messager était largement postérieure au début de l'engraissement anormal [1]. La baisse de l'expression de l'adipsine serait donc une conséquence - pas particulièrement précoce - de l'obćsité, et non pas un marqueur génétique de valcur prédictive.

[1. Dugail I, et al. J Biochem 1990 $265: 1831-3$.]

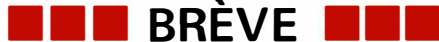

La protéine Tax du virus HTLV-1 pourrait diminuer les capacités de réparation de l'ADN des cellules hôtes. Les proprićtés transactivatrices de la protéine Tax vis-à-vis des propres séquences régulatrices du virus HTLV-1 mais aussi de divers gènes cellulaires comme ceux de l'interleukine 2, du récepteur de l'interlcukinc, des gènes c-fos et GM-CSF (facteur stimulant la formation de colonies de macrophages et de granulocytes) ont été largement démontrées. C'est via l'activation de tels gènes que la protéinc Tax pourrait manifester un potenticl oncogénique, potentiel clairement mis en évidence in vivo, chez des souris transgéniques, où son expression entraîne l'apparition de tumeurs mésenchymateuses. Il faut cependant noter que l'activation des gènes cellulaires précédemment cités est probablement insuffisante pour entraîner la transformation. L'existence dans les ccllules leucémiques adultes de nombreuses anomalics caryotypiques suggérant que des altérations au niveau de l'ADN de l'hôte pouvaicnt concourir à la transformation par le virus HTLV-1, Kuan-Teh Jeang et ses collaborateurs du NIH (Bethesda, MD, USA) ont recherché si l'une des protéines codées par lc virus était capable d'induire des altérations au niveau de l'ADN chromosomique. Un motif identique à celui du site de fixation de la protéine Tax sur le LTR viral ayant été trouvé en amont du site d'initiation de la transcription de la béta polymérase humaine, une enzyme impliquée dans la réparation de l'ADN, les auteurs testèrent les effets de Tax sur l'expression de cette enzyme. Ils apportent la preuve d'un effet réprcsseur de Tax sur l'expression du gène de la béta polymérasc et suggèrent qu'il s'agit d'un des éléments de la cascade des événements induits par l'infection virale.

[Jeang K, et al. Science 1990 ; 247 1082-4.] 
Polyadénylation cytoplasmique et recrutement traductionnel des ARN messagers. L'un des modèles les plus anciennement connus de la régulation traductionnelle, c'est-à-dirc du contrôle de la traduction des ARN, est celui des messagers dormants des ovocytes. Des ARNm sont transcrits au cours de la maturation ovocytaire, mais ne sont pas traduits jusqu'à perception de signaux particulicrs, hormones (progestéronc) ou fécondation. Les ARN, par exemple celui codant pour l'activateur tissulairc du plasminogène chcz le xénopc, sont très peu polyadénylés durant lcur phase de nontraduction, jusqu'à la période durant laquelle l'ovocyte est bloqué après la première division méiotique. La scconde division méiotique suit la stimulation par la progestérone. Cytologiquement, cette activation est marquéc par la dégradation de la mombrane nucléaire; une heure environ après cettc dégradation, les messagers qui doivent être recrutés pour être traduits subissent un important allongcment de leur extension d'acide polyadénylique, nécessaire à leur traduction [1]. Cette polyadénylation, de 150 à 300 résidus $A$, nécessitc la présence de deux séquences à proximité l'une de l'autre, AAUAAA ct UUUUUAU ( $\mathrm{U}=$ acide uridylique). La première de ces séquences cst le motif canonique de polyadénylation présent une vingtaine de bascs avant le site d'addition des résidus A en 3' des messagers cucaryotiqucs. Le second élément semble être caractéristique des messagers qui doivent subir une polyadénylation cytoplasmique pour être activés traductionncllement. De plus, la polyadénylate synthétase active dans le cytoplasme (et donc reconnaissant le motif UUUUUAU) semble différente de l'enzyme catalysant les réactions nucléaircs de polyadénylation [2]. Les mécanismes responsables de l'activation de la traduction après polyadénylation restent inconnus.

[1. Vassali JD, et al. Genes Dev 1989 ; 3: 2163-71.]

[2. Fox CA, et al. Genes Dev 1989 ; 3 : 2151-62.]

$\mathrm{m} / \mathrm{s} n^{\circ} 5$ vol. 6 , mai 90 $\square$ Après l'utilisation de CD4 + soluble dans le SIDA, existe-t-il un avenir pour l'utilisation de "leurres " en chimiothérapie antivirale? La stratégic suivie dans l'utilisation de dérivés solubles de CD4 comme agent bloquant l'infection des lymphocytes et monocytes CD4 ${ }^{+}$ par les virus HIV est simple: il s'agit de saturer les sites de la protéine d'enveloppe (gp 120) reconnaissant lc CD4 membranaire par liaison de formes solubles, évitant ainsi, espère-t-on, la liaison du virus aux membranes des cellules cxprimant CD4. Les résultats à attendre de cette stratégic sont incertains, notamment parce que les virus HIV peuvent pénétrer dans les cellules par d'autres voics que la liaison à CD4. Notamment, les complexes entre des particules virales HIV et des anticorps anti-HIV peuvent se fixer aux réceptcurs pour les fragments $\mathrm{Fc}$ des immunoglobulines ou à ccux des composants du complément. Des équipes des laboratoires Bohringer (Ridgeficld, CT, USA et Viennc, Autriche), associées à une équipe de Boston (MA, USA) viennent de produire par génie génétique une forme soluble de ICAM-1 (intercellular adhesion molecule 1), protéine d'adhérence intercellulaire dont la fonction normale est d'interagir avec la protéine LFA1 (lymphocyte function-associated antigen 1) mais qui est utiliséc comme récepteur par le rhinovirus, responsable de rhinites bénignes. In vitro, ces dérivés solubles bloquent l'infection de cellules en culture par le virus [1]. En l'absence de tests in vivo, l'avenir de la méthode reste cependant incertain. L'utilisation du produit en pulvérisations intranasales pourrait en effet produire une immunisation et une rhinite allergique. De plus, si l'excès de ICAM-1 soluble inhibe l'adhérence normale de leucocytes des fosses nasales à leurs cibles, ce traitement pourrait aussi faciliter la progression d'infections locales. A noter que, comme nous l'avons rapporté très récemment, la même équipc de Bahringer, localisée à Ridgefield, a testé l'efficacité d'anticorps monoclonaux anti-
ICAM-1 sur la réaction inflammatoirc bronchique $\left(\mathrm{m} / \mathrm{s}, n^{\circ} 3\right.$, vol. 6 , p. 310). Les auteurs n'ont manifestement pas osé proposer des inhalations de ICAM-1 soluble chez les asthmatiques (!). En revanche, avec les mêmes réserves que pour ICAM-1, ces anticorps devraient aussi pouvoir être utilisés pour limiter l'infection par le rhinovirus en bloquant les réccptcurs pour le virus (c'est-à-dire les molécules ICAM-1). Il scmble que, normalement, le site d'attachement du virus à ICAM-1 soit enfoui dans un "canyon " [2], situé à la surface virale, et donc soit non accessible à des anticorps... et encore moins, probablement, au complexe ICAM-1/anticorps.

[1. Marlin SD, et al. Nature 1990 ; 344: 70-2.]

[2. Bangham CRM, McMichael AJ Nature 1990 : 344 : 16.]

Cellules pancréatiques exocrines et lymphocytes $T$ cytotoxiques. L'interleukine-4 (IL-4) stimule la croissance et la différcnciation des lymphocytes B ainsi que la maturation de cellules $\mathrm{T}$ cytotoxiques $\mathrm{CD}^{+}$. Plusieurs équipes américaines associées viennent de cloner un $\mathrm{ADNc}$ correspondant à des messagers qui s'accumulent dans des cellules $\mathrm{T}$ cytotoxiques stimulécs par IL-4. La séquence de cet $\mathrm{ADNc}$ a révélé qu'il avait le potentiel de coder pour la lipase de type pancréatique qui apparaît ainsi être exprimée dans deux types de cellules, et deux seulcment : les cellules acineuses du pancréas et des lymphocytes [1]. D'autres protéines de type "pancréatique " ont déjà été incriminées dans la fonction cytolytique des lymphocytes $\mathrm{T}$ : des protéases à sérine [2] ct la phospholipase A2. Il apparaît donc que certains mécanismes communs sont utilisés pour la fonction digestive du pancréas et la fonction cytolytique de cellules $\mathrm{T}$.

[1. Grusby MJ, et al. Cell $1990 ; 60$ : 451-9.]

[2. Goldstein P. médecine/sciences $1989 ; 5$ : 546-53.]
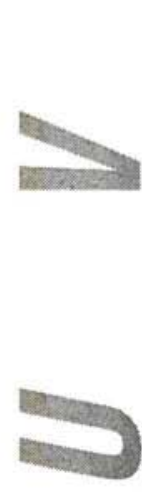
.

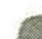

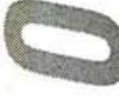

